\title{
Seven Deadly Myths of Testing Moderation in Organizational Research
}

\author{
Jeffrey R. Edwards
}

Moderation is central to research in the organizational and social sciences. Moderation occurs when the relationship between an independent variable and dependent variable depends on the level of a third variable, usually called a moderator variable* (Aiken \& West, 1991; Cohen, 1978). Moderation is involved in research demonstrating that the effects of motivation on job performance are stronger among employees with high abilities (Locke \& Latham, 1990), the effects of distributive justice on employee reactions are greater when procedural justice is low (Brockner \& Wiesenfeld, 1996), and the effects of job demands on illness are weaker when employees have control in their work environment (Karasek, 1979; Karasek \& Theorell, 1990).

Procedures for testing moderation have generated considerable confusion. This confusion is organized here in terms of seven deadly myths. From a research standpoint, these myths are deadly because they lead researchers to make unwise choices, waste time and effort, and draw conclusions that are misleading or incorrect. This chapter describes these seven myths, discusses their basis and origins, and

\footnotetext{
* Throughout this chapter, the term independent variable is synonymous with predictor variable; the term dependent variable is equivalent to criterion variable, outcome variable, and response variable; and the term moderator variable is the same as conditioning variable. In the language of path analysis and structural equation modeling, a dependent variable can be called an endogenous variable, and independent and moderator variables can be either exogenous or endogenous variables, depending on whether they are caused by other variables within a larger model (for the cases examined in this chapter, the independent and moderator variables are treated as exogenous variables).
} 
attempts to dispel them. My goal is not to identify researchers who subscribe to moderation mythology or point to streams of research where the myths run rampant. Rather, my intent is to give researchers good reasons to reject the myths, setting them aside as we pursue answers to important research questions that involve moderation.

\section{The Seven Myths}

Moderation is usually tested with analysis of variance or multiple regression (Cohen, Cohen, West, \& Aiken, 2003; Pedhazur, 1997). Because analysis of variance is a special case of multiple regression (Cohen, 1968), tests of moderation using both approaches are susceptible to essentially the same myths. For simplicity, the present discussion is framed in terms of multiple regression, in which moderation is tested using equations of the following form:

$$
Y=b_{0}+b_{1} X+b_{2} Z+b_{3} X Z+e .
$$

In Equation 6.1, $Y$ is the dependent variable, $X$ is the independent variable, and $Z$ is the moderator variable. The product $X Z$ captures the interaction between $X$ and $Z$ such that, when $X$ and $Z$ are controlled, the coefficient on $X Z$ (i.e., $b_{3}$ ) represents the change in the effect of $X$ on $Y$ for a unit change in $Z$ (Aiken \& West, 1990; Cohen, 1978). The interpretation of $b_{3}$ is symmetric, such that it also indicates the change in the effect of $Z$ on $Y$ for a unit change in $X$. When $Z$ is framed as the moderator variable, it is customary to view $b_{3}$ as the change in the effect of $X$ across levels of $Z$, which is the perspective adopted here.

\section{Myth 1: Product Terms Create Multicollinearity Problems}

Researchers often express the concern that Equation 6.1 is prone to multicollinearity (Morris, Sherman, \& Mansfield, 1986). In general, multicollinearity decreases the stability of regression coefficient estimates and weakens the unique contribution of each predictor to the explained variance in the outcome (Belsley, 1991; Mansfield \& Helms, 1982). Equation 6.1 might seem particularly susceptible to multicollinearity because $X$ and $Z$ can be highly correlated with the 
product term $X Z$. Drawing from Bohrnstedt and Goldberger (1969), when $X$ and $Z$ are normally distributed, the correlation between $X$ and $X Z$ can be written as

$$
r_{X, X Z}=\frac{\mathrm{E}(Z) \mathrm{V}(X)+\mathrm{E}(X) \mathrm{C}(X, Z)}{\sqrt{\mathrm{V}(X)\left[\mathrm{E}(X)^{2} \mathrm{~V}(Z)+\mathrm{E}(Z)^{2} \mathrm{~V}(X)+2 \mathrm{E}(X) \mathrm{E}(Z) \mathrm{C}(X, Z)+\mathrm{V}(X) \mathrm{V}(Z)+\mathrm{C}(X, Z)^{2}\right]}}
$$

where $\mathrm{E}(), \mathrm{V}()$, and $\mathrm{C}()$ are expected value, variance, and covariance operators, respectively. Applying Equation 6.2 to representative values of $X$ and $Z$ demonstrates that $r_{X, X Z}$ can be high. For instance, if $X$ and $Z$ are measured on scales ranging from 1 to 5 and produce means of 3 , unit variances, and a correlation of $.50, \mathrm{r}_{X, X Z}$ would equal .85. Even if $X$ and $Z$ were uncorrelated, $\mathrm{r}_{X, X Z}$ would drop only modestly to .69. For these illustrative values, identical correlations would be obtained for $\mathrm{r}_{Z, X Z}$, the correlation between $Z$ and $X Z$.

Concerns over the correlations of $X Z$ with $X$ and $Z$ have prompted various corrective measures. For instance, Morris et al. (1986) suggested using principal components regression in which $X, Z$, and $X Z$ in Equation 6.1 are replaced with weighted linear composites that exhaust the information in these variables and are uncorrelated with one another. More often, researchers center $X$ and $Z$ at their means, which usually reduces their correlation with $X Z$ (Cronbach, 1987; Jaccard, Wan, \& Turrisi, 1990). This practice capitalizes on the fact the correlation of $X Z$ with $X$ and $Z$ depends on the means of $X$ and $Z$. Returning to Equation 6.2, if $X$ and $Z$ are mean-centered, such that $\mathrm{E}(X)=\mathrm{E}(Z)=0$, the numerator of Equation 6.2 equals zero, which in turn means that $\mathrm{r}_{X, X Z}$ equals zero. Replacing $X$ and $Z$ with one another in Equation 6.2 further shows that when $\mathrm{E}(X)=\mathrm{E}(Z)=$ $0, \mathrm{r}_{Z, X Z}$ also equals zero. When the normality assumption underlying Equation 6.2 is relaxed, $r_{X, X Z}$ and $r_{X Z, X Z}$ do not necessarily equal zero when $X$ and $Z$ are mean-centered, but other values can be derived to center $X$ and $Z$ such that $\mathrm{r}_{X, X Z}$ and $\mathrm{r}_{Z, X Z}$ both equal zero (Bohrnstedt \& Goldberger, 1969; Smith \& Sasaki, 1979).

Although mean-centering usually reduces the correlation of firstorder terms with their products and squares, it has no meaningful effect on the estimation or interpretation of regression equations that contain these terms (Cohen, 1978; Cronbach, 1987; Dunlap \& Kemery, 1987; Kromrey \& Foster-Johnson, 1998). When Equation 6.1 
is estimated, evidence for moderation is obtained from testing $b_{3}$, the coefficient on $X Z$. This test is equivalent to the test of the difference in $R^{2}$ yielded by Equation 6.1 and an equation that drops $X Z$. The $R^{2}$ values for these two equations are insensitive to additive transformations of $X$ and $Z$ (Arnold \& Evans, 1979; Cohen, 1978; Dunlap \& Kemery, 1987), of which mean-centering is a special case. Hence, the test of $b_{3}$ in Equation 6.1 is unaffected by mean-centering, and the "problem" of multicollinearity seemingly indicated by $\mathrm{r}_{X, X Z}$ and $\mathrm{r}_{Z, X Z}$ is more apparent than real. The only real source of multicollinearity in Equation 6.1 involves $r_{X, Z}$, the correlation between $X$ and $Z$, and this correlation is not affected by mean-centering. Unlike $b_{3}$, tests of $b_{1}$ and $b_{2}$ change when $X$ and $Z$ are rescaled, but these changes are not symptoms of multicollinearity or any other analytical anomaly. Rather, they reflect systematic relationships between $b_{1}$ and $b_{2}$ and the scaling of $X$ and $Z$, a topic to which we now turn.

\section{Myth 2: Coefficients on First-Order Terms Are Meaningless}

The interpretation of the coefficients on $X$ and $Z$ when $X Z$ is included in the equation has been a source of confusion. This confusion emanates from the fact that, with $X Z$ in the equation, the coefficients on $X$ and $Z$ are scale-dependent, such that adding or subtracting a constant to $X$ changes the coefficient on $Z$, and vice versa (Cohen, 1978). These effects can be demonstrated using a version of Equation 6.1 that uses $X^{*}=X+\mathrm{c}$ and $Z^{*}=Z+\mathrm{d}$ as predictors, where $\mathrm{c}$ and $\mathrm{d}$ are arbitrary constants:

$$
Y=\mathrm{b}_{0}{ }^{*}+\mathrm{b}_{1}{ }^{*} X^{*}+\mathrm{b}_{2}{ }^{*} Z^{*}+\mathrm{b}_{3}{ }^{*} X^{*} Z^{*}+\mathrm{e} .
$$

The asterisks in Equation 6.3 distinguish the coefficients on $X^{*}, Z$, and $X^{*} Z^{*}$ from those on $X, Z$, and $X Z$ in Equation 6.1. Substituting $X^{*}$ $=X+\mathrm{c}$ and $Z^{*}=Z+\mathrm{d}$ into Equation 6.3 yields

$$
\begin{aligned}
Y & =b_{0}{ }^{*}+b_{1}{ }^{*}(X+\mathrm{c})+b_{2}{ }^{*}(Z+\mathrm{d})+b_{3}{ }^{*}(X+\mathrm{c})(Z+\mathrm{d})+\mathrm{e} \\
& =\mathrm{b}_{0}{ }^{*}+\mathrm{b}_{1}{ }^{*} X+\mathrm{cb}_{1}{ }^{*}+\mathrm{b}_{2}{ }^{*} Z+\mathrm{db}_{2}{ }^{*}+\mathrm{b}_{3}{ }^{*} X Z+\mathrm{db}_{3}{ }^{*} X+\mathrm{cb}_{3}{ }^{*} Z+\mathrm{cdb}_{3}{ }^{*}+\mathrm{e} \\
& =\left(\mathrm{b}_{0}{ }^{*}+\mathrm{cb}_{1}{ }^{*}+\mathrm{db}_{2}{ }^{*}+\mathrm{cdb}_{3}{ }^{*}\right)+\left(\mathrm{b}_{1}{ }^{*}+\mathrm{db}_{3}{ }^{*}\right) X+\left(\mathrm{b}_{2}{ }^{*}+\mathrm{cb}_{3}{ }^{*}\right) Z+\mathrm{b}_{3}{ }^{*} X Z+\mathrm{e} .
\end{aligned}
$$


Equation 6.4 expresses the coefficients on $X, Z$, and $X Z$ in terms of the coefficients from Equation 6.3 and the constants $\mathrm{c}$ and d. Comparing Equations 6.1 and 6.4 shows that $\mathrm{b}_{0}=\mathrm{b}_{0}{ }^{*}+\mathrm{cb}_{1}{ }^{*}+\mathrm{db}_{2}{ }^{*}+\mathrm{cdb}_{3}{ }^{*}$, $b_{1}=b_{1}{ }^{*}+d_{3}{ }^{*}, b_{2}=b_{2}{ }^{*}+c_{3}{ }^{*}$, and $b_{3}=b_{3}{ }^{*}$. Solving these expressions for $b_{0}{ }^{*}, b_{1}{ }^{*}, b_{2}{ }^{*}$, and $b_{3}{ }^{*}$ and substituting the results into Equation 6.3 reveals how the rescaling $\mathrm{X}$ and $\mathrm{Z}$ changes the coefficients produced by Equation 6.1 (Arnold \& Evans, 1979; Cohen, 1978):

$Y=\left(b_{0}-c_{1}-d_{2}+c d b_{3}\right)+\left(b_{1}-d_{3}\right) X^{\star}+\left(b_{2}-c_{3}\right) Z^{\star}+b_{3} X^{*} Z^{\star}+e$.

Equation 6.5 shows that, when $X$ and $Z$ are rescaled by adding $c$ and $d$, respectively, $b_{1}$ is reduced by $d_{3}, b_{2}$ is reduced by $c_{3}$, and $b_{3}$ is unaffected. In addition, the intercept $b_{0}$ is reduced by $c b_{1}$ and $d b_{2}$ and increased by $\mathrm{cdb}_{3}$, although these changes do not alter the form of the interaction indicated by Equation 6.5.

The effects of rescaling $X$ and $Z$ have caused concern because, in organizational and social research, measures of $X$ and $Z$ are usually at the interval level. For such measures, adding or subtracting an arbitrary constant is a permissible transformation from a statistical standpoint. However, this transformation changes the magnitude, sign, and significance of the coefficients on $X$ and $Z$. Consequently, some researchers have declared the coefficients on $X$ and $Z$ "arbitrary nonsense" (Cohen, 1978, p. 861) and assert that attempts to test or interpret these coefficients are "useless" (Allison, 1977, p. 148).

Although the effects of rescaling $X$ and $Z$ are undeniable, these effects do not render the coefficients on $X$ and $Z$ arbitrary or useless. Rather, rescaling $X$ and $Z$ changes their coefficients in systematic ways that can facilitate interpretation. This point is rooted in the principle that, when a regression equation contains $X, Z$, and $X Z$, the coefficient on $X$ is the slope of $Y$ on $X$ when $Z$ equals zero (Aiken \& West, 1991). This principle is seen by rewriting Equation 6.1 in terms of simple slopes (Aiken \& West, 1991; Jaccard et al., 1990):

$$
Y=\left(b_{0}+b_{2} Z\right)+\left(b_{1}+b_{3} Z\right) X+e .
$$

When $Z=0$, the term $b_{3} Z=0$, and the compound coefficient on $X$ reduces to $b_{1}$. This principle is symmetric, such that $b_{2}$ is the slope of $Y$ on $Z$ when $X$ equals zero. This principle also applies to Equation 6.3, such that $\mathrm{b}_{1}{ }^{*}$ is the slope of $Y$ on $X^{*}$ when $Z^{*}=0$, and $\mathrm{b}_{2}^{*}$ is 
the slope of $Y$ on $Z^{*}$ when $X^{*}=0$. Hence, whether rescaling $X$ and $Z$ yields useful coefficients depends on whether $X^{*}=0$ and $Z^{*}=0$ are meaningful values. If rescaling $X$ and $Z$ shifts their distribution such that $X^{*}=0$ and $Z^{*}=0$ fall outside the data, then $b_{1}{ }^{*}$ and $b_{2}{ }^{*}$ are not meaningful because they estimate slopes at points that do not exist in the data. On the other hand, if rescaling locates $X^{*}=0$ and $Z^{*}=$ 0 within the bounds of the data, then $b_{1}{ }^{*}$ and $b_{2}{ }^{*}$ can be interpreted accordingly. For instance, if $\mathrm{c}$ and $\mathrm{d}$ are the negative of the means of $X$ and $Z$, respectively, such that $X^{*}$ and $Z^{*}$ are mean-centered versions of $X$ and $Z$, then $\mathrm{b}_{1}{ }^{*}$ is the slope of $Y$ on $X$ at the mean of $Z$, and $b_{2}{ }^{*}$ is the slope of $Y$ on $Z$ at the mean of $X . X$ and $Z$ can be rescaled using other values, such as the negative of scores representing one standard deviation above and below the means of $X$ and $Z$, which can help clarify the form of the interaction captured by $X Z$ (Aiken \& West, 1991; Jaccard et al., 1990) Alternately, Equation 6.1 can be estimated, and Equation 6.5 can be used to calculate coefficients that would be obtained for different rescalings of $X$ and $Z$. Hence, the coefficients on $X$ and $Z$ are meaningful and useful when $X=0$ and $Z=0$ are within the range of the data, and these coefficients are needed to compute simple slopes that clarify the form of the moderating effect (Aiken \& West, 1991; Jaccard et al., 1990).

\section{Myth 3: Measurement Error Poses Little Concern When First-Order Terms Are Reliable}

Researchers tend to underemphasize the effects of measurement error on estimates obtained from Equation 6.1. This tendency is manifested in two ways. First, concerns over measurement error typically hinge on whether reliability estimates meet some conventional threshold, such as usual .70 criterion for Cronbach's alpha (Lance, Butts, \& Michels, 2006). If the threshold is met, analyses proceed as if the effects of measurement error can be disregarded. Second, reliability estimates are usually reported for $X$ and $Z$ but not the product term $X Z$. This practice implies that if $X$ and $Z$ exhibit adequate reliabilities, the reliability of $X Z$ is likewise adequate. Because the reliability of $X Z$ is not computed, its effects on the estimation of Equation 6.1 can easily escape the attention of researchers.

The effects of measurement error on tests of moderation deserve greater attention than they are usually accorded. Methodological 
work has shown that measurement error drastically reduces the power to detect moderator effects (Aiken \& West, 1991; Arnold, 1982; Busemeyer \& Jones, 1983; Dunlap \& Kemery, 1988; Jaccard \& Wan, 1995; Lubinski \& Humphreys, 1990), and this problem does not disappear when the reliabilities of $X$ and $Y$ exceed .70. Moreover, the reliability of the product term $X Z$ can be quite low even when the reliabilities of $X$ and $Z$ might be considered adequate. Drawing from Bohrnstedt and Marwell (1978), when $X$ and $Z$ follow a bivariate normal distribution, the reliability of $X Z$ can be expressed as

$$
\rho_{X Z}=\frac{\mathrm{E}(X)^{2} \mathrm{~V}(Z) \rho_{Z}+\mathrm{E}(Z)^{2} \mathrm{~V}(X) \rho_{X}+2 \mathrm{E}(X) \mathrm{E}(Z) \mathrm{C}(X, Z)+\mathrm{C}(X, Z)^{2}+\mathrm{V}(X) \mathrm{V}(Z) \rho_{X} \rho_{Z}}{\mathrm{E}(X)^{2} \mathrm{~V}(Z)+\mathrm{E}(Z)^{2} \mathrm{~V}(X)+2 \mathrm{E}(X) \mathrm{E}(Z) \mathrm{C}(X, Z)+\mathrm{C}(X, Z)^{2}+\mathrm{V}(X) \mathrm{V}(Z)}
$$

where $\rho_{X Z}$ is the reliability of $X Z$ and $\rho_{X}$ and $\rho_{Z}$ are the reliabilities of $X$ and $Z$, respectively. To illustrate how $\rho_{X Z}$ relates to $\rho_{X}$ and $\rho_{Z}$, consider the case in which $X$ and $Z$ are standardized, such that Equation 6.7 simplifies to

$$
\rho_{X Z}=\frac{r_{X Z}^{2}+\rho_{X} \rho_{Z}}{r_{X Z}^{2}+1}
$$

where $r_{X Z}$ is the correlation between $X$ and $Z$. Using Equation 6.8, if $X$ and $Z$ are uncorrelated and have reliabilities of .70, the reliability of $X Z$ equals .49. If the correlation between $X$ and $Z$ is .25, the reliability of $X Z$ equals .52 , and if the correlation increases further to .50 , the reliability of $X Z$ becomes .59 . As these examples show, even when the reliabilities of $X$ and $Z$ meet conventional standards, the reliability of $X Z$ can fail to reach those very same standards.

It should be noted that the reliability of $X Z$ is scale-dependent, such that adding a constant to $X$ or $Z$ will change the reliability of $X Z$ (Bohrnstedt \& Marwell, 1978). These scaling effects operate through $\mathrm{E}(X)$ and $\mathrm{E}(Z)$, which appear in the numerator and denominator of Equation 6.7. Because $\rho_{X Z}$ depends on the scales of $X$ and $Z$, Bohrnstedt and Marwell (1978) cautioned against estimating $\rho_{X Z}$ when $X$ and $Z$ are measured on interval scales, noting that the estimated value of $\rho_{X Z}$ is as arbitrary as the origins of $X$ and $Z$. However, when discussing the reliability of $X Z$, Lubinski and Humphreys (1990) surmised that the scaling effects of $X$ and $Z$ on $\rho_{X Z}$ can be disregarded when the test of $X Z$ controls for $X$ and $Z$, because the increment in $R^{2}$ explained by $X Z$ is unaffected by the scaling of $X$ and $Z$ (Arnold 
\& Evans, 1979; Cohen, 1978; Dunlap \& Kemery, 1987). It follows that the reduction in statistical power created by measurement error in $X, Z$, and $X Z$ is unaffected by the scaling of $X$ and $Z$. Perhaps the reliability of the partialed $X Z$ product is not affected by rescaling $\mathrm{X}$ and $Z$, even though the reliability of the $X Z$ product itself is scaledependent. This issue could be clarified by deriving the reliability of the partialed $X Z$ product, using the work of Bohrnstedt and Marwell (1978) as a starting point.

\section{Myth 4: Product Terms Should Be Tested Hierarchically}

Studies of moderation often test the interaction term $X Z$ hierarchically, first estimating an equation using only $X$ and $Z$ as predictors and then estimating an equation that adds $X Z$, as in Equation 6.1. The difference in $R^{2}$ between these two equations is then tested using the following $F$-ratio or its equivalent (e.g., Pedhazur, 1997):

$$
F=\frac{R_{X, Z, X Z}^{2}-R_{X, Z}^{2}}{\left(1-R_{X, Z, X Z}^{2}\right) /(N-4)}
$$

where $R_{X, Z}^{2}$ is the $R^{2}$ from the equation using $X$ and $Z$ as predictors, $R^{2}{ }_{X, Z, X Z}$ is the $R^{2}$ from the equation that adds $X Z$ to $X$ and $Z$ as predictors, and $N$ is the sample size. The $F$-ratio given in Equation 6.9 has 1 numerator degree of freedom and $\mathrm{N}-4$ denominator degrees of freedom. A statistically significant $F$-ratio is taken as evidence of moderation. This hierarchical approach to testing moderation is firmly rooted in the literature, as evidenced by methodological discussions that refer to moderated regression as "hierarchical" (Busemeyer \& Jones, 1983; Cortina, 1993; Jaccard et al., 1990; Lubinski \& Humphreys, 1990) and present separate regression equations with and without the $X Z$ product term (Arnold, 1982; Arnold \& Evans, 1979; Cortina, 1993; Dunlap \& Kemery, 1988; Jaccard et al., 1990; Lubinski \& Humphreys, 1990; MacCallum \& Mar, 1995; Morris et al., 1986; Zedeck, 1971).

The hierarchical approach to testing moderation has two drawbacks. First, when a moderating effect is captured by a single product term, such as $X Z$ in Equation 6.1, hierarchical analysis is unnecessary because the $F$-ratio in Equation 6.9 will give the same result as the $t$ test of the coefficient on XZ (Cohen, 1978; Jaccard et al., 1990; 
Kromrey \& Foster-Johnson, 1998; McClelland \& Judd, 1993). If the increment in $R^{2}$ explained by the moderating effect is of interest, it can be computed by squaring the $t$-statistic to obtain the corresponding $F$-statistic and multiplying this quantity by the denominator of Equation 6.9. When a moderating effect involves more than one product term, as in ANOVA designs with factors that have more than two levels, it might be convenient to test the effect and compute the increment in $R^{2}$ using the hierarchical approach, although the same result is given by procedures that test simultaneous constraints on regression coefficients, such as the GLM procedure of SPSS using the LMATRIX subcommand.

A second drawback of the hierarchical approach is that it can generate interpretations of the coefficients on $X$ and $Z$ that are misleading. In practice, researchers who use the hierarchical approach often interpret the coefficients on $X$ and $Z$ at the first step, before $X Z$ has been added to the equation. These interpretations are unconditional, such that the effect of $X$ on $Y$ is treated as constant across levels of $Z$, and likewise, the effect of $Z$ on $Y$ is viewed as constant across levels of $X$. However, if the coefficient on $X Z$ is significant in the second step, then the effects of $X$ and $Z$ on $Y$ are both conditional, such that the effect of each variable depends on the level of the other variable. The conditional effect of $X$ is shown by Equation 6.6, in which the coefficient on $X$ is the compound term $\left(b_{1}+b_{3} Z\right)$. Rewriting Equation 6.6 to show the conditional effect of $Z$ yields the compound coefficient $\left(b_{2}+b_{3} X\right)$ on $Z$. Hence, when the second step indicates that moderation exists, the coefficients on $X$ and $Z$ in the first step should be disregarded because, by definition, moderation means that the effects of $X$ and $Z$ on $Y$ are not each represented by a single value, but by a range of values that vary across levels of the other variable. This variation is not captured by the coefficients on $X$ and $Z$ from the first step, and reporting these coefficients invites their interpretation, which is unwarranted when the second step gives support for moderation.

\section{Myth 5: Curvilinearity Can Be Disregarded When Testing Moderation}

Studies of moderation rarely examine the squared terms $X^{2}$ and $Z^{2}$ along with the product term $X Z$. Disregarding $X^{2}$ and $Z^{2}$ might seem justified for various reasons. For instance, if an interaction between 
$X$ and $Z$ is predicted on theoretical grounds, then testing $X^{2}$ and $Z^{2}$ would go beyond what was predicted and might be frowned upon as atheoretical (Shepperd, 1991). In a similar vein, researchers simply might not consider curvilinear effects as often as moderating effects (Cortina, 1993; Ganzach, 1997). This possibility is consistent with a PsycINFO search of articles published since 1980 in the Academy of Management Journal, the Journal of Applied Psychology, and Organizational Behavior and Human Decision Processes. Of these articles, 232 mentioned the terms moderation or moderated in the title or abstract, whereas only 34 mentioned the terms curvilinear or quadratic. Researchers might also avoid testing $X^{2}$ and $Z^{2}$ along with $X Z$ due to interpretational difficulties. Methodological discussions of moderation and curvilinearity usually treat $X Z, X^{2}$, and $Z^{2}$ as separate terms, each with its own interpretation (Cortina, 1993; Ganzach, 1997, 1998; Lubinski \& Humphreys, 1990; MacCallum \& Mar, 1995). Any difficulties involved in the interpretation of $X Z$ are likely to be compounded when $X^{2}$ and $Z^{2}$ are added to the picture.

As a general rule, researchers investigating moderation hypotheses should consider testing $X^{2}$ and $Z^{2}$ along with $X Z$ (Cortina, 1993; Ganzach, 1997; Lubinski \& Humphreys, 1990; MacCallum \& Mar, 1995) Doing so helps establish that the coefficient on $X Z$ taken as evidence for moderation does not spuriously reflect curvilinearity associated with $X^{2}, Z^{2}$, or both. Results for $X Z$ can be misleading because, when $X$ and $Z$ are correlated, $X Z$ is usually correlated with $X^{2}$ and $Z^{2}$ (Cortina, 1993; Ganzach, 1997; Lubinski \& Humphreys, 1990; MacCallum \& Mar, 1995). Drawing from Bohrnstedt and Goldberger (1969), if $X$ and $Z$ are normally distributed with zero means, the correlation between $X Z$ and $X^{2}$ is

$$
r_{X Z, X^{2}}=\frac{2 \mathrm{~V}(X) \mathrm{C}(X, Z)}{\sqrt{2 \mathrm{~V}(X)^{2}\left[\mathrm{~V}(X) \mathrm{V}(Z)+\mathrm{C}(X, Z)^{2}\right]}} .
$$

Inserting representative values of $\mathrm{V}(X), \mathrm{V}(Z)$, and $\mathrm{C}(X, Z)$ into Equation 6.10 shows how the correlation between $X^{2}$ and $X Z$ is influenced by the association between $X$ and $Z$. For instance, when $X$ and $Z$ are uncorrelated, $\mathrm{C}(X, Z)=0$, and $X Z$ and $X^{2}$ are also uncorrelated. As the correlation between $X$ and $Z$ increases, the correlation between $X Z$ and $X^{2}$ likewise increases. Shifting the means of $X$ and $Z$ from 
zero alters the correlation between $X Z$ and $X^{2}$, but these changes do not affect tests of $X Z$ and $X^{2}$ when $X$ and $Z$ are controlled. These principles also apply to the correlation between $X Z$ and $Z^{2}$, which can be computed by reversing the positions of $X$ and $Z$ in Equation 6.10.*

Simulation work has examined the effects of controlling for $X^{2}$ and $Z^{2}$ along with $X$ and $Z$ when testing $X Z$. When $X^{2}$ and $Z^{2}$ are uncorrelated with $X Z$, controlling for $X^{2}$ with $Z^{2}$ guards against inferring support for moderation that actually reflects curvilinearity, with a reduction in statistical power limited to the degrees of freedom consumed by $X^{2}$ with $Z^{2}$ (Cortina, 1993). When $X^{2}$ and $Z^{2}$ are correlated with $X Z$, the effect size of $X Z$ is reduced when $X^{2}$ and $Z^{2}$ are controlled, because $X^{2}$ and $Z^{2}$ account for a portion of the variance that would be explained by $X Z$ (Ganzach, 1998). This reduction in effect size increases the risk of Type II error for the test of $X Z$ (Kromrey-Foster \& Johnson, 1999) On the other hand, when $X^{2}$ and $Z^{2}$ are not controlled, the risk of Type I error for testing $X Z$ can increase, given that moderation can be inferred when curvilinearity is actually responsible for the variance explained by $X Z$. The relative risks of Type I and Type II errors for tests of $X Z$ also depend on the signs of the coefficients on $X Z, X^{2}$, and $Z^{2}$ (Ganzach, 1997). On balance, the benefits of controlling for $X^{2}$ and $Z^{2}$ seem to outweigh the costs (Cortina, 1993; Ganzach, 1997, 1987; Lubinski \& Humphreys, 1990; MacCallum \& Mar, 1995). Naturally, if curvilinear effects for $X^{2}$ and $Z^{2}$ are not predicted a priori as hypotheses that compete with the moderating effect of $X Z$, results for $X^{2}$ and $Z^{2}$ should be considered tentative, pending cross-validation (Kromrey-Foster \& Johnson, 1999; Shepperd, 1991).

Examining $X^{2}$ and $Z^{2}$ might also be reasonable from a conceptual standpoint. Strictly speaking, most theories in the organizational and social sciences predict relationships that are monotonic rather than linear (Busemeyer \& Jones, 1983; Ganzach, 1998). Hypotheses of the form "if $X$ increases, $Y$ will increase" do not stipulate that the relationship between $X$ and $Y$ is linear, but instead make the more modest claim that higher values of $X$ are associated with higher values of $Y$. In some instances, a monotonic relationship such as this

* The correlation between $X^{2}$ and $Z^{2}$ can also be derived from Bohrnstedt and Goldberger (1969), and again this correlation is a function of the correlation between $X$ and $Z$. 
might be better conceived as curvilinear rather than linear, as illustrated by the diminishing effects of income on happiness (Eckersley, 2000). Alternately, if a linear relationship is hypothesized, analyzing curvilinear terms verifies that the relationship was not, in fact, curvilinear, yielding a stronger test of the hypothesis. Tests of curvilinearity involving $X^{2}$ and $Z^{2}$ can benefit from including $X Z$, given that the correlations among $X Z, X^{2}$, and $Z^{2}$ can generate misleading evidence for curvilinearity as well as moderation (Ganzach, 1997).

Finally, difficulties associated with interpreting $X Z, X^{2}$, and $Z^{2}$ separately can be addressed by interpreting these terms jointly along with $X$ and $Z$. This task can be approached by drawing from the logic used to interpret simple slopes in moderated regression analysis (Aiken \& West, 1991). This logic is illustrated by Equation 6.6, which rearranges terms in Equation 6.1 to show the effect of $X$ on $Y$ at various levels of $Z$. This logic can be extended to an equation that includes $X, Z, X Z, X^{2}$, and $Z^{2}$, as given below:

$$
Y=b_{0}+b_{1} X+b_{2} Z+b_{3} X Z+b_{4} X^{2}+b_{5} Z^{2}+e .
$$

Rewriting Equation 6.11 to show the relationship between $X$ and $Y$ at various levels of $Z$ yields

$$
Y=\left(b_{0}+b_{2} Z+b_{5} Z^{2}\right)+\left(b_{1}+b_{3} Z\right) X+b_{4} X^{2}+e .
$$

Equation 6.12 is a quadratic function relating $X$ to $Y$ that depends on the level of $Z$. The curvature of the function, indicated by $b_{4}$, remains constant across levels of $Z$, whereas the intercept and the coefficient on $X$ are influenced by $Z$, as shown by the terms $\left(b_{0}+b_{2} Z+b_{5} Z^{2}\right)$ and $\left(b_{1}+b_{3} Z\right)$, respectively. For a quadratic function such as Equation 6.12 , the coefficient on $\mathrm{X}$ is the slope of the function at the point $X=$ 0 , as can be seen by taking the derivative of $Y$ with respect to $X$ :

$$
d Y / d X=\mathrm{b}_{1}+\mathrm{b}_{3} Z+2 \mathrm{~b}_{4} X .
$$

Equation 6.13 gives the instantaneous slope of $Y$ on $X$. When $X=0$, Equation 6.13 reduces to $b_{1}+b_{3} Z$, which is the coefficient on $X$ in Equation 6.12.

Results for Equation 6.12 can be interpreted as follows. If $b_{4}$ is positive, the function relating $X$ to $Y$ is curved upward, as shown in the top three panels of Figure 6.1. If $\left(b_{1}+b_{3} Z\right)$ is negative, the curve 

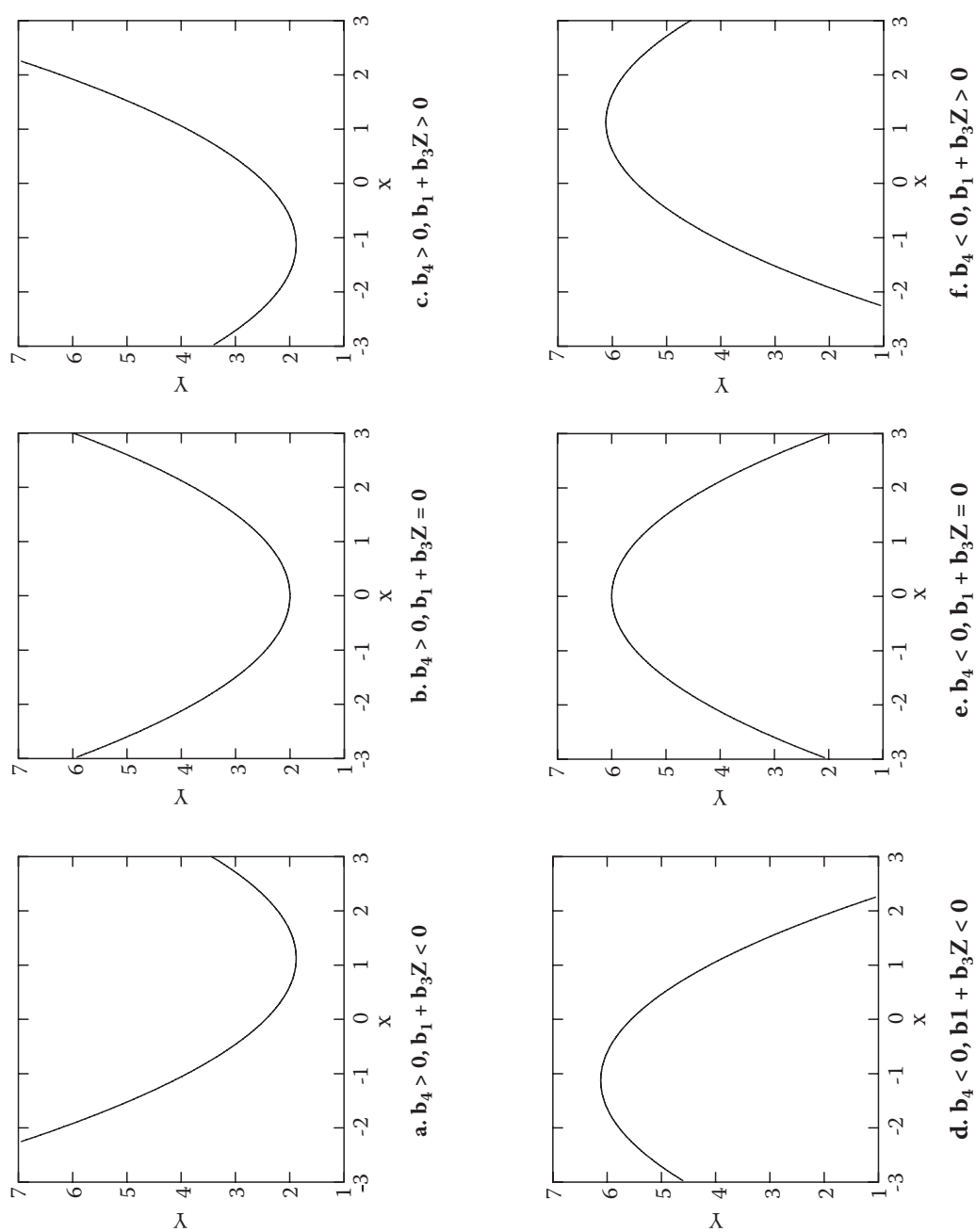

Figure 6.1 Quadratic functions relating $X$ to $Y$ for different values of $b_{4}$ and $\mathrm{b}_{1}+\mathrm{b}_{3} Z$. 
is negatively sloped at $X=0$, which means that the minimum of the curve is shifted to the right, as in Figure 6.1a. If $\left(b_{1}+b_{3} Z\right)$ is positive, the curve is positively sloped at $X=0$, and the minimum of the curve is shifted to the left, as in Figure 6.1c. If $\left(b_{1}+b_{3} Z\right)$ equals zero, the curve is flat at $X=0$, and the minimum of the curve is centered at $X$ $=0$, as in Figure 6.1b. In contrast, if $b_{4}$ is negative, the function relating $X$ to $Y$ is curved downward, as in the bottom three panels of Figure 6.1. If $\left(b_{1}+b_{3} Z\right)$ is negative, the curve is again negatively sloped at $X=0$, which now means that the maximum of the curve is shifted to the left, as in Figure 6.1d. If $\left(b_{1}+b_{3} Z\right)$ is positive, the curve is again positively sloped at $X=0$, which means the maximum of the curve is shifted to the right, as in Figure 6.1f. Finally, if $\left(b_{1}+b_{3} Z\right)$ equals zero, the curve is again flat at $X=0$, which indicates that the maximum of the curve is centered at $X=0$, as in Figure 6.1e.

The foregoing discussion leads to the following interpretation of the coefficients on $X Z, X^{2}$, and $Z^{2}$ in Equation 6.11. Specifically, the coefficient on $X Z$ is part of the compound term that indicates the slope of the function relating $X$ to $Y$ at the point $X=0$, and the coefficient on $X^{2}$ represents the curvature of the function. Together, the coefficients on $X^{2}$ and $X Z$ capture the curvature and horizontal location, respectively, of the function relating $X$ to $Y$. The coefficient on $Z^{2}$ is part of the intercept and indicates whether the effect of $Z$ on the intercept varies across levels of $Z$. Hence, the coefficient on $Z^{2}$ should be considered if the vertical position of the function relating $X$ to $Y$ is relevant from a conceptual standpoint. Additional guidelines for interpreting curvilinear relationships between $X$ and $Y$ are provided by Aiken and West (1991), and these guidelines can be applied to the curvilinear function in Equation 6.12. An alternative approach to interpreting Equation 6.11 frames the relationship between $X, Z$, and $Y$ as a three-dimensional surface, of which the functions in Figure 6.1 are cross sections at selected levels of $Z$ (Edwards, 2002; Edwards \& Parry, 1993). This approach is useful when the joint effects of $X$ and $Z$ on $Y$ are framed in terms of fit, similarity, or agreement.

\section{Myth 6: Product Terms Can Be Treated as Causal Variables}

In studies of moderation, the product term $X Z$ is sometimes treated as a causal variable. This practice is common in studies that examine 
whether the moderating effect captured by $X Z$ is mediated by some other variable, or what has been termed mediated moderation (Baron \& Kenny, 1986). Mediated moderation is frequently examined using a version of the causal steps procedure that assesses the change in the coefficient on $X Z$ that results when a mediator variable is added to Equation 6.1 (Baron \& Kenny, 1986; Muller, Judd, \& Yzerbyt, 2005). The first step of the procedure is to test $b_{3}$ in Equation 6.1 to verify that a moderating effect exists. In the second step, the following equation is estimated:

$$
M=\mathrm{b}_{0}+\mathrm{b}_{1} X+\mathrm{b}_{2} Z+\mathrm{b}_{3} X Z+\mathrm{e}
$$

where $M$ is the mediating variable through which the moderating effect is hypothesized to be transmitted. The second step requires that $b_{3}$ is significant, meaning that $X Z$ is related to $M$. The third and fourth steps involve the following equation:

$$
Y=\mathrm{b}_{0}+\mathrm{b}_{1} X+\mathrm{b}_{2} Z+\mathrm{b}_{3} X Z+\mathrm{b}_{4} M+\mathrm{e} .
$$

Equation 6.15 adds $M$ to Equation 6.1 as an additional predictor of $Y$. The third step is to test $\mathrm{b}_{4}$ to verify that the $M$ is related to $Y$, such that the mediator is related to the outcome. Finally, in the fourth step, $b_{3}$ is examined to determine whether it is smaller in Equation 6.15 than in Equation 6.1. If $b_{3}$ in Equation 6.15 is reduced to the point it is not significant, then it is concluded that $M$ fully mediates the effect of $X Z$ on $Y$. If $\mathrm{b}_{3}$ is smaller but remains significant, then $M$ is viewed as a partial mediator of the effect of $X Z$ on $Y$.

The treatment of $X Z$ as a causal variable is misguided, because $X Z$ has no causal potency in its own right. Rather, $X Z$ is merely a mathematical device that captures the extent to which the effect of $X$ on $Y$ varies across levels of $Z$ (or, equivalently, whether the effect of $Z$ on $Y$ varies across levels of $X$ ). It is $X$ and $Z$, the variables that constitute $X Z$, that are capable of influencing $Y$ in a causal sense. The product $X Z$ does not represent some unique entity that exists separately from $X$ and $Z$, and therefore it cannot exert an effect on $Y$ beyond that generated by $X$ and $Z$. The role of $X Z$ in causal models relating $X, M$, and $Z$ to $Y$ can be examined by expressing causal paths in terms of simple slopes that show how the paths vary across levels of $Z$ (Edwards \& Lambert, 2007). In this manner, the variables with causal potency are properly depicted, and the manner in which product terms such as $X Z$ alter the relationships among these variables is clarified. 
Myth 7: Testing Moderation in Structural

Equation Modeling Is Impractical

In organizational and social research, studies of moderation rely almost exclusively on ANOVA or regression analysis. These methods rest on the assumption that the variables involved in the analysis are measured without error (Berry, 1993; Pedhazur, 1997). When this assumption is violated, results are affected in various ways. In particular, measurement error in the dependent variable biases $R^{2}$ estimates downward. Measurement error in the independent variables can bias coefficient estimates upward or downward, depending on the pattern and degree of measurement error. The biasing effects of measurement error can alter the substantive conclusions drawn from analyses of moderation and further aggravate the problems of reduced statistical power discussed earlier.

The effects of measurement error can be addressed using structural equation modeling with latent variables (Bollen, 1989; Kline, 2004). Although structural equation modeling usually involves linear relationships among latent variables, methods have been developed to estimate moderating effects (Cortina, Chen, \& Dunlap, 2001; Jaccard \& Wan, 1995; Jöreskog \& Yang, 1996; Kenny \& Judd, 1984; Kline \& Moosbrugger, 2000; Li, Harmer, Duncan, Duncan, Acock, \& Boles, 1998; Ping, 1996; Schumacker \& Marcoulides, 1998). These methods have been under development for over two decades, yet they are rarely applied in studies of moderation in the organizational and social sciences. When the methods are acknowledged, they are usually set aside as too complex or impractical due to limitations of available estimation procedures. For instance, structural equation modeling typically relies on maximum likelihood estimation, which incorporates the assumption that observed variables follow a multivariate normal distribution (Bollen, 1989). This assumption is violated when product terms are analyzed, because a product term is not normally distributed even when the variables that constitute the product are normally distributed. Products of observed variables are used in most methods for analyzing moderation in structural equation modeling (Cortina et al., 2001; Kenny \& Judd, 1984; Li et al., 1998; Marsh, Wen, \& Hau, 2004), which means that these methods violate a key assumption of maximum likelihood estimation. For these and other reasons, the use of structural equation modeling in tests of moderation is all but nonexistent. 
When compared to ANOVA or regression analysis, methods for analyzing moderation using structural equation modeling are undeniably more complex. However, these methods have become increasingly accessible in recent years, as a result of tutorials that demystify the methods (Cortina et al., 2001) and the availability of syntax for applying the methods in published work (Cortina et al., 2001; Li et al., 1998; Schumacker \& Marcoulides, 1998). In addition, analytical developments have addressed estimation issues associated with moderated structural equation models. For instance, violations of multivariate normality can be addressed using methods that adjust standard errors and chi-square estimates (Chou, Bentler, \& Satorra, 1991) or by applying the bootstrap, which can be used to generate sampling distributions for parameter estimates without assuming normality (Bollen \& Stine, 1992; Efron \& Tibshirani, 1993; Mooney \& Duval, 1993). These methods have proven effective in structural equation modeling (Curran, West, \& Finch, 1996; Nevitt \& Hancock, 2001) and show promise in models that include moderation (Yang-Wallentin \& Jöreskog, 2001). Hence, methodological advancements in moderated structural equation modeling are ongoing, and researchers interested in testing moderation should incorporate these advancements into their work. The benefits of applying these methods will be worth the effort, given the detrimental effects of measurement error on tests of moderation.

\section{Myths Beyond Moderation}

The seven myths described here focused on studies of moderation. However, these myths apply to other analytical procedures that involve transformations of independent variables. For instance, studies that examine curvilinear relationships between $X$ and $Y$ typically use powers of $X$, such as $X^{2}, X^{3}$, and so forth, to represent curvilinearity (Cohen, 1978). Many of the myths associated with tests of moderation using the product term $X Z$ apply to tests of curvilinearity using $X^{2}, X^{3}$, and higher powers of $X$, given that raising $X$ to a power is equivalent to using product terms created by multiplying $X$ by itself. Moreover, just as tests of moderation can benefit from incorporating curvilinearity, tests of curvilinearity can be sharpened by incorporating moderation (Ganzach, 1997; Lubinski \& Humphreys, 1990). In addition, studies that examine fit, similarity, and agreement often 
use the absolute or squared difference between two variables as a predictor, sometimes along with the two variables that constitute the difference. Such studies have fallen victim to the myths discussed here, and the problems generated by the myths are compounded by those associated with difference scores (Edwards, 1994; Johns, 1981), which have spawned myths of their own (Edwards, 2001). Thus, the myths discussed here extend beyond studies of moderation, and researchers would be well advised to guard against these myths in studies of curvilinearity, fit, similarity, agreement, and other phenomena that involve transformations of independent variables.

\section{Conclusion}

The seven myths summarized here are prevalent in organizational and social research that examines moderation. Although some of the myths might have a kernel of truth, each myth has the capacity to lead researchers astray, invest unnecessary effort, and draw conclusions that are misleading or incorrect. By raising awareness to these myths, it is hoped that researchers will avoid the myths in their own work and point them out when they surface in the work of students and colleagues. Doing so will help increase the quality of research that involves moderation, leading to better answers to important theoretical and substantive questions that we collectively pursue.

\section{References}

Aiken, L. A., \& West, S. G. (1991). Multiple regression: Testing and interpreting interactions. Newbury Park, CA: Sage.

Allison, P. D. (1977). Testing for interaction in multiple regression. American Journal of Sociology, 83, 144-153.

Arnold, H. J. (1982). Moderator variables: A clarification of conceptual, analytic, and psychometric issues. Organizational Behavior and Human Performance, 29, 143-174.

Arnold, H. J., \& Evans, M. G. (1979). Testing multiplicative models does not require ratio scales. Organizational Behavior and Human Performance, 24, 41-59.

Baron, R. M., \& Kenny, D. A. (1986). The moderator-mediator variable distinction in social psychological research: Conceptual, strategic, and statistical considerations. Journal of Personality and Social Psychology, 51, 1173-1182. 
Belsley, D. A. (1991). Conditioning diagnostics: Collinearity and weak data in regression. New York: Wiley.

Berry, W. D. (1993). Understanding regression assumptions. Newbury Park, CA: Sage.

Bohrnstedt, G. W., \& Goldberger, A. S. (1969). On the exact covariance of products of random variables. Journal of the American Statistical Association, 64, 1439-1442.

Bohrnstedt, G. W., \& Marwell, G. (1978). The reliability of products of two random variables. In K. F. Schuessler (Ed.), Sociological methodology (pp. 254-273). San Francisco: Jossey-Bass.

Bollen, K. A. (1989). Structural equations with latent variables. New York: Wiley.

Bollen, K. A., \& Stine, R. A. (1992). Bootstrapping goodness-of-fit measures in structural equation models. Sociological Methods \& Research, 21, 205-229.

Brockner, J., \& Wiesenfeld, B. M. (1996). An integrative framework for explaining reactions to decisions: Interactive effects of outcomes and procedures. Psychological Bulletin, 120, 189-208.

Busemeyer, J. R., \& Jones, L. E. (1983). Analysis of multiplicative combination rules when the causal variables are measured with error. Psychological Bulletin, 93, 549-562.

Chou, C. P., Bentler, P. M., \& Satorra, A. (1991). Scaled test statistics and robust standard errors for non-normal data in covariance structure analysis: A Monte Carlo study. British Journal of Mathematical and Statistical Psychology, 44, 347-357.

Cohen, J. (1968). Multiple regression as a general data-analytic system. Psychological Bulletin, 70, 426-443.

Cohen, J. (1978). Partialed products are interactions: Partialed powers are curve components. Psychological Bulletin, 85, 858-866.

Cohen, J., Cohen, P., West, S. G., \& Aiken, L. S. (2003). Applied multiple regression/correlation analysis for the behavioral sciences (3rd ed.). Mahwah, NJ: Erlbaum.

Cortina, J. M. (1993). Interaction, nonlinearity, and multicollinearity: Implications for multiple regression. Journal of Management, 19, 915-922.

Cortina, J. M., Chen, G., \& Dunlap, W. P. (2001). Testing interaction effects in LISREL: Examination and illustration of available procedures. Organizational Research Methods, 4, 324-360.

Cronbach, L. J. (1987). Statistical tests for moderator variables: Flaws in analyses recently proposed. Psychological Bulletin, 102, 414-417.

Curran, P. J., West, S. G., \& Finch, J. F. (1996). The robustness of test statistics to nonnormality and specification error in confirmatory factor analysis. Psychological Methods, 1, 16-29. 
Dunlap, W. P., \& Kemery, E. R. (1987). Failure to detect moderating effects: Is multicollinearity the problem? Psychological Bulletin, 102, 418-420.

Dunlap, W. P., \& Kemery, E. R. (1988). Effects of predictor intercorrelations and reliabilities on moderated multiple regression. Organizational Behavior and Human Decision Process, 41, 248-258.

Eckersley, R. (2000). The mixed blessings of material progress: Diminishing returns in the pursuit of happiness. Journal of Happiness Studies, 1, 267-292.

Edwards, J. R. (1994). The study of congruence in organizational behavior research: Critique and a proposed alternative. Organizational Behavior and Human Decision Processes, 58, 51-100 (erratum, 58, 323-325).

Edwards, J. R. (2001). Ten difference score myths. Organizational Research Methods, 4, 264-286.

Edwards, J. R. (2002). Alternatives to difference scores: Polynomial regression analysis and response surface methodology. In F. Drasgow \& N. W. Schmitt (Eds.), Advances in measurement and data analysis (pp. 350-400). San Francisco: Jossey-Bass.

Edwards, J. R., \& Lambert, L. S. (2007). Methods for integrating moderation and mediation: A general analytical framework using moderated path analysis. Psychological Methods, 12, 1-22.

Edwards, J. R., \& Parry, M. E. (1993). On the use of polynomial regression equations as an alternative to difference scores in organizational research. Academy of Management Journal, 36, 1577-1613.

Efron, B., \& Tibshirani, R. (1993). An introduction to the bootstrap. New York: Chapman \& Hall.

Ganzach, Y. (1997). Misleading interaction and curvilinear terms. Psychological Methods, 2, 235-247.

Ganzach, Y. (1998). Nonlinearity, multicollinearity, and the probability of Type II error in detecting interaction. Journal of Management, 24, 615-622.

Jaccard, J., \& Wan, C. K. (1995). Measurement error in the analysis of interaction effects between continuous predictors using multiple regression: Multiple indicator and structural equation approaches. Psychological Bulletin, 117, 348-357.

Jaccard, J., Wan, C. K., \& Turrisi, R. (1990). The detection and interpretation of interaction effects between continuous variables in multiple regression. Multivariate Behavioral Research, 25, 467-478.

Johns, G. (1981). Difference score measures of organizational behavior variables: A critique. Organizational Behavior and Human Performance, 27, 443-463. 
Jöreskog, K. G., \& Yang, F. (1996). Nonlinear structural equation models: The Kenny-Judd model with interaction effects. In G. A. Marcoulides \& R. E. Schumacker (Eds.), Advanced structural equation modeling (pp. 57-88). Hillsdale, NJ: Erlbaum.

Karasek, R. A., Jr. (1979). Job demands, job decision latitude, and mental strain: Implications for job redesign. Administrative Science Quarterly, 24, 285-308.

Karasek, R. A., \& Theorell, T. (1990). Healthy work: Stress, productivity, and the reconstruction of working life. New York: Basic Books.

Kenny, D. A., \& Judd, C. M. (1984). Estimating the nonlinear and interactive effects of latent variables. Psychological Bulletin, 96, 201-210.

Klein, A., \& Moosbrugger, H. (2000). Maximum likelihood estimation of latent interaction effects with the LMS method. Psychometika, 65, 457-474.

Kline, R. B. (2004). Principles and practice of structural equation modeling (2nd ed.). New York: Guilford Press.

Kromrey, J. D., \& Foster-Johnson, L. (1998). Mean centering in moderated multiple regression: Much ado about nothing. Educational and Psychological Measurement, 58, 42-68.

Kromrey, J. D., \& Foster-Johnson, L. (1999). Statistically differentiating between interaction and nonlinearity in multiple regression analysis: A Monte Carlo investigation of a recommended strategy. Educational and Psychological Measurement, 59, 392-413.

Lance, C. E., Butts, M. M., \& Michels, L. C. (2006). The sources of four commonly reported cutoff criteria: What did they really say? Organizational Research Methods, 9, 202-220.

Li, F., Harmer, P., Duncan, T. E., Duncan, S. C., Acock, A., \& Boles, S. (1998). Approaches to testing interaction effects using structural equation modeling methodology. Multivariate Behavioral Research, 33, 1-39.

Locke, E. A., \& Latham, G. P. (1990). A theory of goal setting and task performance. Englewood Cliffs, NJ: Prentice Hall.

Lubinski, D., \& Humphreys, L. G. (1990). Assessing spurious "moderator effects": Illustrated substantively with the hypothesized ("synergistic") relation between spatial and mathematical ability. Psychological Bulletin, 107, 385-393.

MacCallum, R. C., \& Mar, C. M. (1995). Distinguishing between moderator and quadratic effects in multiple regression. Psychological Bulletin, 118, 405-421.

Mansfield, E. R., \& Helms, B. P. (1982). Detecting multicollinearity. The American Statistician, 36, 158-160.

Marsh, H. W., Wen, Z., \& Hau, K.-T. (2004). Structural equation models of latent interactions: Evaluation of alternative estimation strategies and indicator construction. Psychological Methods, 9, 275-300. 
McClelland, G. H., \& Judd, C. M. (1993). Statistical difficulties of detecting interactions and moderator effects. Psychological Bulletin, 114, 376-390.

Mooney, C. Z., \& Duval, R. D. (1993). Bootstrapping: A nonparametric approach to statistical inference. Newbury Park, CA: Sage.

Morris, J. H., Sherman, J. D., \& Mansfield, E. R. (1986). Failures to detect moderating effects with ordinary least squares-moderated multiple regression: Some reasons and a remedy. Psychological Bulletin, 99, 282-288.

Muller, D., Judd, C. M., \& Yzerbyt, V. Y. (2005). When moderation is mediated and mediation is moderated. Journal of Personality and Social Psychology, 89, 852-863.

Nevitt, J., \& Hancock, G. R. (2001). Performance of bootstrapping approaches to model test statistics and parameter standard error estimation in structural equation modeling. Structural Equation Modeling, 8, 353-377.

Pedhazur, E. J. (1997). Multiple regression in behavioral research (3rd ed.). New York: Holt.

Ping, R. A., Jr. (1996). Latent variable interaction and quadratic effect estimation: A two-step techniques using structural equation analysis. Psychological Bulletin, 119, 166-175.

Schumacker, R. E., \& Marcoulides, G. A. (Eds.). (1998). Interaction and nonlinear effects in structural equation modeling. Hillsdale, NJ: Erlbaum.

Shepperd, J. A. (1991). Cautions in assessing spurious "moderator effects." Psychological Bulletin, 110, 315-317.

Smith, K. W., \& Sasaki, M. S. (1979). Decreasing multicollinearity: A method for models with multiplicative functions. Sociological Methods and Research, 8, 296-313.

Yang-Wallentin, F., \& Jöreskog, K. G. (2001). Robust standard errors and chi-squares for interaction models. In G. A. Marcoulides \& R. E. Schumacker (Eds.), New developments and techniques in structural equation modeling (pp. 159-171). Mahwah, NJ: Erlbaum.

Zedeck, S. (1971). Problems with the use of "moderator" variables. Psychological Bulletin, 76, 295-310. 